\title{
Muscle MRI findings in a one-year-old girl with merosin-deficient congenital muscular dystrophy type 1A due to LAMA2 mutation: A case report
}

\author{
YINGYIN LIANG $^{1 *}$, GUIDIAN LI $^{2 *}$, SONGLIN CHEN ${ }^{1}$, RONGXING HE$^{2}$, XIANGXUE ZHOU $^{1}$, \\ YINGMING CHEN $^{2}, \mathrm{XUE} \mathrm{XU}^{1}$, RONGLAN ZHU ${ }^{1}$ and CHENG ZHANG ${ }^{1}$ \\ Departments of ${ }^{1}$ Neurology and ${ }^{2}$ Radiology, The First Affiliated Hospital of \\ Sun Yat-Sen University, Guangzhou, Guangdong 510700, P.R. China
}

Received June 9, 2017; Accepted June 19, 2017

DOI: $10.3892 /$ br.2017.935

\begin{abstract}
The objective of the present study was to characterize the muscle magnetic resonance imaging (MRI) features of a 1-year-old girl with merosin-deficient congenital muscular dystrophy type 1A (MDC1A). Beginning as an infant, this patient exhibited severe hypotonia and proximal weakness, as well as delays in developmental milestones. Her serum creatine kinase levels at 3 months, 8 months and 1 year were 2,959, 1,621 and 1,659 U/1, respectively. Brain MRI indicated symmetric, mild T1WI low, mild T2WI and FLAIR high radial patterns in the white matter of the Cornu posterius of the ventricular lateral. Gene sequencing demonstrated a heterozygous frame-shift mutation in the LAMA2 gene, consisting of an AG deletion at nucleotides 2049-2050 (LAMA2 c.2049_2050delAG). Lower limb muscle MRI presented obvious fatty infiltration of the muscles and muscle atrophy during the early stage of the disease. The gluteus maximus, erector spinae, vastus intermedius, vastus lateralis, adductor magnus, soleus and gastrocnemius muscles were involved, whereas the piriformis, obturator internus, pectineus, adductor longus, adductor brevis and sartorius muscles presented mild or no involvement. Fatty infiltration of the erector spinae was observed during the early stage of the disease. As an additional tool in the differential diagnosis of muscle disorders, muscle MRI can delay the need for muscle biopsy.
\end{abstract}

\section{Introduction}

Merosin-deficient congenital muscular dystrophy type 1A (MDC1A) is characterized by early onset in infancy of

Correspondence to: Dr Yingyin Liang, Department of Neurology, The First Affiliated Hospital of Sun Yat-Sen University, Guangzhou, Guangdong 510700, P.R. China

E-mail: lyyin@mail.sysu.edu.cn

\section{* Contributed equally}

Key words: merosin-deficient congenital muscular dystrophy type $1 \mathrm{~A}$, muscle magnetic resonance imaging critical muscle weakness, high creatine kinase (CK) levels, and diffuse white matter changes in the brain. Being noninvasive, muscle magnetic resonance imaging (MRI) is useful in the diagnosis and follow-up of patients with MDC1A. Because MDC1A is characterized by changes in white matter, most patients with this disease are analyzed by brain MRI. To the best of the authors' knowledge, there are few studies of patients with MDC1A that have involved muscle MRI. This report describes a patient with MDC1A who was analyzed by muscle MRI, and reviews studies summarizing characteristics of muscle MRI in patients with MDC1A.

\section{Case report}

A 1-year-old girl, born at gestational age 39 weeks and 5 days by natural labor, presented with a 1-year history of central hypotonia and proximal weakness on March 27, 2015, when admitted to hospital (The First Affiliated Hospital of Sun Yat-Sen University, Guangzhou, China). As an infant, she had a weak cry and floppy limbs. Her developmental milestones were delayed; she first raised her head at the age of 8 months and first sat independently at the age of 9 months. However, she was unable to hold her head while sitting and has been unable to stand or walk, even with the help of her parents. Her intellectual and speech development was normal, and her family history was negative for this or a similar condition.

The study protocol was approved by the Ethics Committee of Sun Yat-Sen University (Guangzhou, China), and the patient's family provided written informed consent for publication of the present study.

Physical examination. Physical examination presented myopathic facies and a single crease of the right palm, the latter also observed in her father. Her intellectual and language function was normal. She could sit without support but could not walk or stand, and her neck was too floppy for her to hold her head up while sitting. No muscle atrophy or pseudohypertrophy was observed. Both her upper and lower limbs reported hypotonia and weakness, which was more proximal than distal and predominant in her shoulder and pelvic girdle. Muscle assessment using the standard Medical 
Research Council scale (grade 0-5) indicated that muscle strength of her four limbs from proximal to distal was $3 / 5,3 / 5$, $4 / 5$ and $4 / 5$, respectively (1). Sensory examination could not be completed due to patient noncooperation. Her tendon reflexes and Babinski's sign were negative.

Serum CK levels. Her serum CK levels at ages 3 and 8 months and one year were 2,959,1,621 and 1,659 U/1, respectively. At age 1 year, her blood ammonia concentration was $73.4 \mathrm{mmol} / \mathrm{l}$ and her resting state blood lactic acid concentration was $1.3 \mathrm{mmol} / \mathrm{l}$. Urine and blood screenings for hereditary metabolic diseases were unremarkable.

Ultrasound cardiography. Ultrasound cardiography reported patent foramen ovale at age 1 day, but normal results at age 1 year.

MRI. Brain MRI performed at age 3 months presented symmetrical, mild T1WI low, mild T2WI and FLAIR high radial pattern signals in the white matter of the Cornu posterius of the ventricular lateral. The cortices, ventricles, cerebellum and basal ganglia were normal.

Muscle MRI performed at age 1 year presented wide spread abnormalities in the muscle groups of the lower legs, including thigh and pelvis degeneration, infiltration and fiber edema. The bilateral posterior tibial, soleus, gastrocnemius and anterior tibial muscles reported abnormal patterns, including high signal intensity on T1WI and T2WI and isointensity or hyperintensity on T2WI-SPAIR. Among the lower leg muscles, the soleus revealed T2WI-SPAIR hyperintensity (Fig. 1A-1, A-2 and A-3). The bilateral vastus internus, vastus medialis, vastus lateralis, rectus femoris, biceps femoris, adductor magnus, semitendinosus and semimembranosus muscles were atrophied, with T1WI and T2WI showing isointensity or hyperintensity. In contrast, T2WI-SPAIR presented isointensity of some muscles, whereas others, including the vastus medialis and vastus lateralis muscles, showed hyperintensity (Fig. 1B-1, B-2 and B-3). The bilateral gluteus maximus, gluteus medius and gluteus minimus muscles were atrophied with T1WI, T2WI and T2WI-SPAIR muscle MRI presenting pinnate patterns of abnormal hyperintensity (Fig. 1C-1, C-2 and C-3). The bilateral erector spinae muscles showed hyperintensity on T1WI and T2WI and isointensity on T2WI-SPAIR (Fig. 1D-1, D-2 and D-3).

Fatty infiltration of muscles was assessed by the Mercuri scale, as modified by Fischer et al (2) (Fig. 1). A total of 28 muscles were assessed, including four lower leg muscles (gastrocnemius, soleus, posterior tibial and anterior tibial), 11 thigh muscles (adductor magnus, gracilis, adductor longus, sartorius, rectus femoris, vastus internus, vastus medialis, vastus lateralis, biceps femoris, semitendinosus and semimembranosus) and 13 pelvis muscles (gluteus maximus, gluteus medius, gluteus minimus, iliopsoas, piriformis, obturator internus, adductor brevis, obturator externus, tensor fasciaelatae, pectineus, erector spinae, psoas major and iliacus). Six muscles (piriformis, obturator internus, pectineus, adductor longus, adductor brevis and sartorius) were identified to be grade 0 , eight (iliopsoas, psoas major, iliacus, rectus femoris, semitendinosus, semimembranosus, gracilis and posterior tibial) were grade 1, four (gluteus minimus, obturator externus, vastus medialis and biceps femoris) were grade 2 , ten (gluteus maximus, gluteus medius, tensor fasciae latae, erector spinae, vastus lateralis, vastus internus, adductor magnus, gastrocnemius, soleus and anterior tibial) were grade 3 , and none were grade 4 .

Molecular genetic testing. Genomic DNA from the patient was extracted from peripheral blood leukocytes and high-throughput sequencing (3-5) was performed to test abnormalities in 63 genes associated with neuromuscular disorders. The patient was found to have a heterozygous frame-shift mutation in the LAMA2 gene, in which the nucleotides AG at positions 2049-2050were deleted (LAMA2 c.2049_2050delAG), resulting in early protein termination [p.(Arg683fs)]. Multiplex polymerase chain reaction of the LAMA2 gene of her parents' DNA indicated that her father had the same mutation, whereas her mother was normal. These findings confirmed the diagnosis of congenital MDC1A.

\section{Discussion}

MDC1A, originally described in 1994 (6), was shown to result from mutations in the laminin- $\alpha 2$ gene (LAMA2), which encodes the extracellular matrix protein merosin. Most mutations detected to date have been nonsense and splicing site point mutations and frame shift mutations (7). The absence of merosin results in early weakness, delayed development milestones, high blood levels of CK and normal cognition with white matter abnormalities on brain MRI $(8,9)$.

The present study encountered a 1-year old girl who presented with congenital hypotonia, delayed motor milestones and weakness. Muscle MRI revealed fatty infiltration and edema of selected muscles. Testing of myodystrophy genes confirmed the diagnosis of MDC1A. Muscle MRI, a noninvasive method of assessing the extent of muscle damage, has become a very useful tool in the diagnosis and follow-up of patients with muscle diseases, including muscular dystrophy, inflammatory myopathies and metabolic myopathies $(10,11)$. Muscle MRI may be under-utilized in patients with MDC1A.

Similar to the MRI manifestations of other types of muscular dystrophy (12-14), fatty infiltration is the major abnormal pattern observed in MDC1A. The patient demonstrated obvious fatty infiltration into muscles of the pelvis, thigh and lower leg. The muscles most commonly involved during the early stages of disease included the gluteus maximus, gluteus medius, erector spinae, proximal vastus intermedius, proximal vastus lateralis, adductor magnus, soleus, anterior tibial and gastrocnemius muscles (15). On T1WI, these muscles had a fatty infiltration grade of 3 . The T1WI fatty infiltration grade was higher for the proximal than the distal quadriceps femoris, being grades 2-3 for the proximal and grades 1-2 for the distal vastus intermedius, vastus lateralis and vastus medialis muscles. Involvement of the piriformis, obturator internus, pectineus, adductor longus, adductor brevis and sartorius muscles was relatively uncommon until the terminal stage of disease. In the patient, the vastus intermedius, vastus lateralis, vastus medialis and rectus femoris muscles had fatty infiltration grades of 3, 3,2 and 1, respectively. The vastus intermedius demonstrated earliest involvement, followed by the vastus lateralis and medialis muscles. Similar radiological 

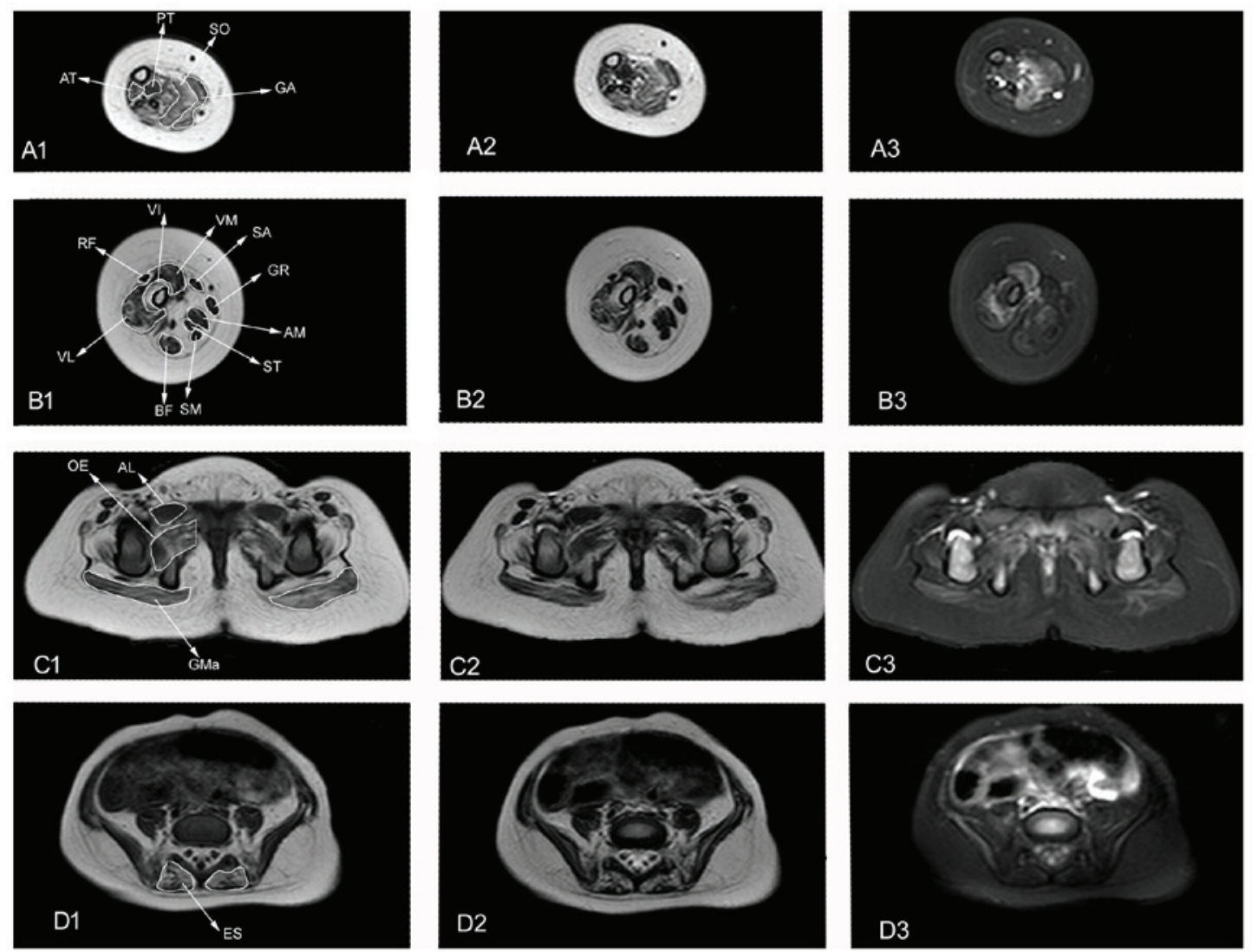

Figure 1. Muscle MRI of the patient at 1 year of age. Axial T1WI, T2WI and T2WI SPAIR images of the lower leg, thigh and pelvis. (A) 1-3: Lower leg muscle MRI presenting PT, SO, GA, and AT fatty infiltration and edema. (B) 1-3: Thigh muscle MRI presenting VI, VM, VL, BF, AM, ST and SB atrophy and fatty infiltration. VM and VL present edema. (C) 1-3 and (D) 1-3: Pelvis muscle MRI presenting GMa atrophy (C1-3); GMa, OE and ES fatty infiltration (C1-3, D 1-3); and GMa, AL and OE edema (C1-3, D 1-3). MRI, magnetic resonance imaging; WI, weighted image; PT, posterior tibial; SO, soleus; GA, gastrocnemius; AT, anterior tibial; VI, vastus internus; VM, vastus medialis; SA, sartorius; GR, gracilis; AM, adductor magnus; ST, semitendinosus; SM, semimembranosus; BF, biceps femoris; VL, vastus lateralis; RF, rectus femoris; GMa, gluteus maximus; GP, greater psoas; OE, obturator externus; $\mathrm{AL}$, adductor longus; $\mathrm{ES}$, erector spinae.

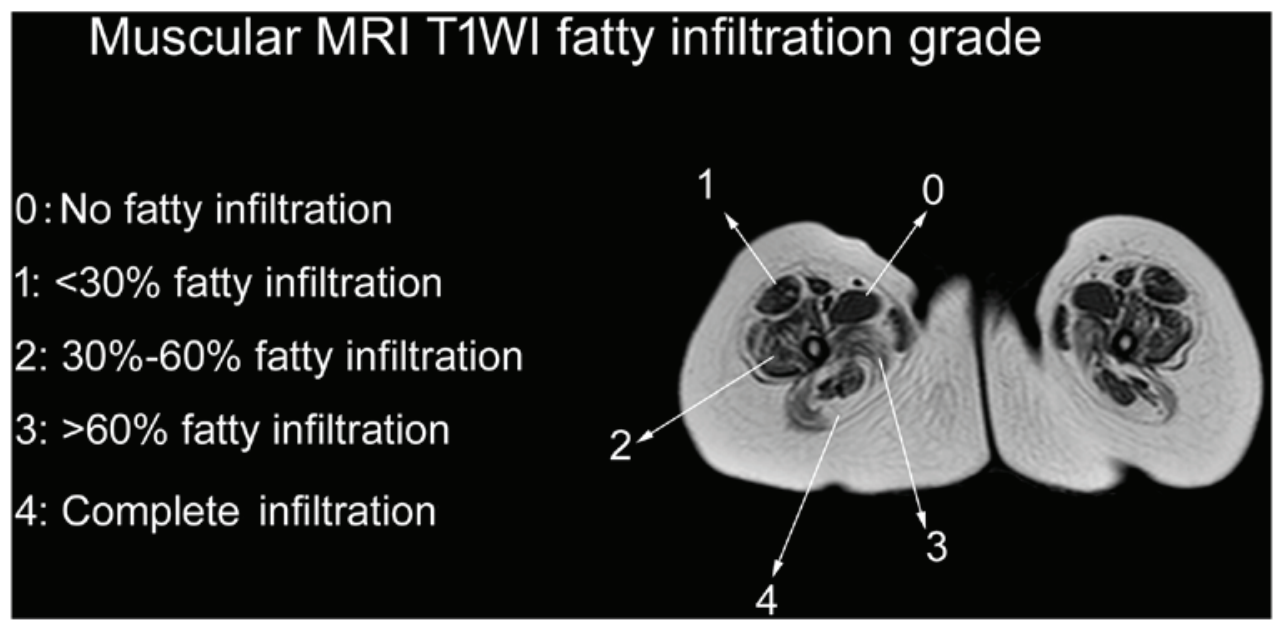

Figure 2. Thigh slice image of muscular MRI T1WI fatty infiltration, showing grades 0-4, as assessed by the Mercuri scale modified by Fischer. MRI, magnetic resonance imaging; WI, weighted image.

features have been observed in patients with COL6 Ullrich and laminopathy, which is caused by mutations in genes encoding extracellular matrix proteins (15). The tested patient presented involvement of the erector spinae muscle at the onset of MDC1A (Fig. 2D-1, D-2 and D-3) with a fatty infiltration grade of 3 on T1WI. Involvement of paraspinal muscles at the onset of disease has been observed in patients with other muscle diseases, including rigid spine muscular dystrophy and congenital muscular dystrophy and spinal rigidity $(16,17)$. In contrast to Duchenne muscular dystrophy, 
pseudohypertrophy $(18,19)$ is not commonly observed during the progression of MDC1A. Rather, muscular atrophy was indicated to occur early in the patient, including atrophy of the gluteus maximus, quadriceps femoris, biceps, adductor magnus, semitendinosus and semimembranosus muscles.

MDC1A is the most common form of congenital muscular dystrophy. Most patients with MDC1A are too young to undergo muscle biopsy. Muscle MRI, an additional tool in the differential diagnosis of muscle disorders, can help patients delay muscle biopsy. The findings in our patient indicate that the muscle MRI features of MDC1A include: i) Fatty infiltration as the major pattern; ii) common involvement of the gluteus maximus, erector spinae, vastus intermedius, vastus lateralis, adductor magnus, soleus and gastrocnemius muscles; iii) mild or no involvement of the piriformis, obturator internus, pectineus, adductor longus, adductor brevis and sartorius muscles; iv) fatty infiltration into the erector spinae during the early stage of disease; and v) onset of muscle atrophy at the onset of the disease. Additional study is required, including of patients at different clinical stages.

\section{References}

1. Paternostro-Sluga T, Grim-Stieger M, Posch M, Schuhfried O, Vacariu G, Mittermaier C, Bittner C and Fialka-Moser V: Reliability and validity of the Medical Research Council (MRC) scale and a modified scale for testing muscle strength in patients with radial palsy. J Rehabil Med 40: 665-671, 2008.

2. Fischer D, Kley RA, Strach K, Meyer C, Sommer T, Eger K, Rolfs A, Meyer W, Pou A, Pradas J, et al: Distinct muscle imaging patterns in myofibrillar myopathies. Neurology 71 : 758-765, 2008

3. Li H and Durbin R: Fast and accurate long-read alignment with Burrows-Wheeler transform. Bioinformatics 26: 589-595, 2010.

4. Zhang L, Zhang J, Yang J, Ying D, Lau YL and Yang W: PriVar: a toolkit for prioritizing SNVs and indels from next-generation sequencing data. Bioinformatics 29: 124-125, 2013.

5. Yang Y, Muzny DM, Reid JG, Bainbridge MN, Willis A, Ward PA, Braxton A, Beuten J, Xia F, Niu Z, et al: Clinical whole-exome sequencing for the diagnosis of mendelian disorders. N Engl J Med 369: 1502-1511, 2013.

6. Tomé FM, Evangelista T, Leclerc A, Sunada Y, Manole E, Estournet B, Barois A, Campbell KP and Fardeau M: Congenital muscular dystrophy with merosin deficiency. C R AcadSci III 317: 351-357, 1994.

7. Xiong H, Tan D, Wang S, Song S, Yang H, Gao K, Liu A, Jiao H, Mao B, Ding J, et al: Genotype/phenotype analysis in Chinese laminin- $\alpha 2$ deficient congenital muscular dystrophy patients. Clin Genet 87: 233-243, 2015.
8. Fardeau M, Tomé FM, Helbling-Leclerc A, Evangelista T, Ottolini A, Chevallay M, Barois A, Estournet B, Harpey JP, Fauré S, et al: Congenital muscular dystrophy with merosin deficiency: Clinical, histopathological, immunocytochemical and genetic analysis. Rev Neurol (Paris) 152: 11-19, 1996 (In French).

9. Incecik F, Herguner OM, Ceylaner S and Altunbasak S: Merosinnegative congenital muscular dystrophy: Report of five cases. J Pediatr Neurosci 10: 346-349, 2015.

10. Peters SA, Kohler C, Schara U, Hohendahl J, Vorgerd M, Nicolas V and Heyer CM: Museular magnetic resonance imaging for evaluation of myopathies in children. Klin Padiatr 220: 37-46, 2007.

11. Cejas CP, Serra MM, Galvez DFG, Cavassa EA, Taratuto AL, Vazquez GA, Massaro MEL and Schteinschneider AV: Muscle MRI in pediatrics: Clinical, pathological and genetic correlation. Pediatr Radiol 47: 724-735, 2017.

12. Díaz-Manera J, Llauger J, Gallardo E and Illa I: Muscle MRI in muscular dystrophies. Acta Myol 34: 95-108, 2015.

13. Polavarapu K, Manjunath M, Preethish-Kumar V, Sekar D, Vengalil S, Thomas P, Sathyaprabha TN, Bharath RD and Nalini A: Muscle MRI in Duchenne muscular dystrophy: Evidence of a distinctive pattern. Neuromuscul Disord 26: 768-774, 2016.

14. Fatehi F, Salort-Campana E, Le Troter A, Bendahan D and Attarian S: Muscle MRI of facioscapulohumeral dystrophy (FSHD): A growing demand and a promising approach. Rev Neurol (Paris) 172: 566-571, 2016.

15. Harris E, McEntagart M, Topf A, Lochmüller H, Bushby K, Sewry $\mathrm{C}$ and Straub V: Clinical and neuroimaging findings in two brothers with limb girdle muscular dystrophy due to LAMA2 mutations. Neuromuscul Disord 27: 170-174, 2017.

16. Mercuri E, Clements E, Offiah A, Pichiecchio A, Vasco G, Bianco F, Berardinelli A, Manzur A, Pane M, Messina S, et al: Muscle magnetic resonance imaging involvement in muscular dystrophies with rigidity of the spine. Ann Neurol 67: 201-208, 2010.

17. Flanigan KM, Kerr L, Bromberg MB, Leonard C, Tsuruda J, Zhang P, Gonzalez-Gomez I, Cohn R, Campbell KP and Leppert M: Congenital muscular dystrophy with rigid spine syndrome: A clinical, pathological, radiological, and genetic study. Ann Neurol 47: 152-161, 2000.

18. Godi C, Ambrosi A, Nicastro F, Previtali SC, Santarosa C, Napolitano S, Iadanza A, Scarlato M, Natali Sora MG, Tettamanti A, et al: Longitudinal MRI quantification of muscle degeneration in Duchenne muscular dystrophy. Ann Clin Transl Neurol 3: 607-622, 2016.

19. Vohra RS, Lott D, Mathur S, Senesac C, Deol J, Germain S, Bendixen R, Forbes SC, Sweeney HL, Walter GA, et al: Magnetic resonance assessment of hypertrophic and pseudo-hypertrophic changes in lower leg muscles of boys with Duchenne muscular dystrophy and their relationship to functional measurements. PLoS One 10: $\mathrm{e} 0128915,2015$. 